\title{
Polarization properties of localized structures in VCSELs
}

\author{
Etienne Averlant ${ }^{\mathrm{a}, \mathrm{b}}$, Mustapha Tlidi ${ }^{\mathrm{a}}$, Thorsten Ackemann ${ }^{\mathrm{c}}$, Hugo Thienpont ${ }^{\mathrm{b}}$, and Krassimir \\ Panajotov $\mathrm{b}, \mathrm{d}$ \\ ${ }^{a}$ Faculté des Sciences, Université libre de Bruxelles, Boulevard du triomphe, Brussels, Belgium \\ ${ }^{\mathrm{b}}$ Brussels Photonics Team (B-PHOT), Pleinlaan 2, Brussels, Belgium \\ ${ }^{\mathrm{c}} \mathrm{SUPA}$ and Department of Physics, University of Strathclyde, Glasgow, United Kingdom \\ ${ }^{\mathrm{d} I n s t i t u t e}$ of Solid State Physics, 72 Tzarigradsko Chaussee Boulevard, 1784 Sofia, Bulgaria
}

\begin{abstract}
Broad area Vertical-Cavity Surface-Emitting Lasers (VCSELs) have peculiar polarization properties which are a field of study by itself. ${ }^{1-3}$ These properties have already been used for localized structure generation, in a simple configuration, where only one polarization component was used. ${ }^{4}$ Here, we present new experimental and theoretical results on the complex polarization behavior of localized structures generated in an optically-injected broad area VCSEL. A linear stability analysis of the spin-flip VCSEL model is performed for the case of broad area devices, in a restrained and experimentally relevant parameter set. Numerical simulations are performed, in one and two dimensions. They reveal existence of vector localized structures. These structures have a complex polarization state, which is not simply a linear polarization following the one of the optical injection. Experimental results confirm theoretical predictions. Applications of this work can lead to the encoding of small color images in the polarization state of an ensemble of localized structures at the surface of a broad area VCSEL.
\end{abstract}

Keywords: VCSEL, Cavity Solitons, Localized Structures, spatially extended systems, polarization

\section{INTRODUCTION}

Nowadays, practically all information-related technologies rely on photonics. This can certainly be explained using economical and/or historical perpectives, but the unique properties of light definitely played a great role in its importance in this application field.

One can, by example, make use of the polarization properties of light to multiplex signals: two orthogonal polarization components can carry a different signal along their propagation, using the very same polarization maintaining optical fiber or path in air, at the same time.

Photonics is also appealing for applications in information related technologies because of the variety of possibilities offered by the nonlinear interaction between light and matter. Using this interaction can allow to generate pulses that keep their temporal or spatial shape during their propagation. These pulses are called solitons. When a soliton is generated inside a cavity, it is called a Cavity Soliton (CS). This object is a nonlinear dissipative structure that occurs in various fields of nonlinear science (see recent overviews on this issue ${ }^{5-7}$ ). They have been proposed very early to be used as pixels for information transmission and storage. ${ }^{8-11}$ In this idea, the surface of a laser is used as a screen, on which a pixel-map made of spatial CSs is drawn.

However, up to now, this application could possibly not be realized, as a pixel does contain (color) information in a three parameter space, whereas the only information contained in a CS is the fact that it exists or not (a bit). It is hence possible to realise a bitmap using CSs, but, as far as we know, no pixel-map.

A better understanding of CS's polarization could help reaching this multiplexing goal. We published recently a note ${ }^{12}$ about that topic in a Vertical-Cavity Surface-Emitting Laser (VCSEL) submitted to linearly polarized optical injection with a varying polarization angle. The experimental results were described using the VCSEL spin-flip model, ${ }^{13,14}$ to which diffraction was added.

Send correspondence to E.A.: eaverlan@ulb.ac.be 


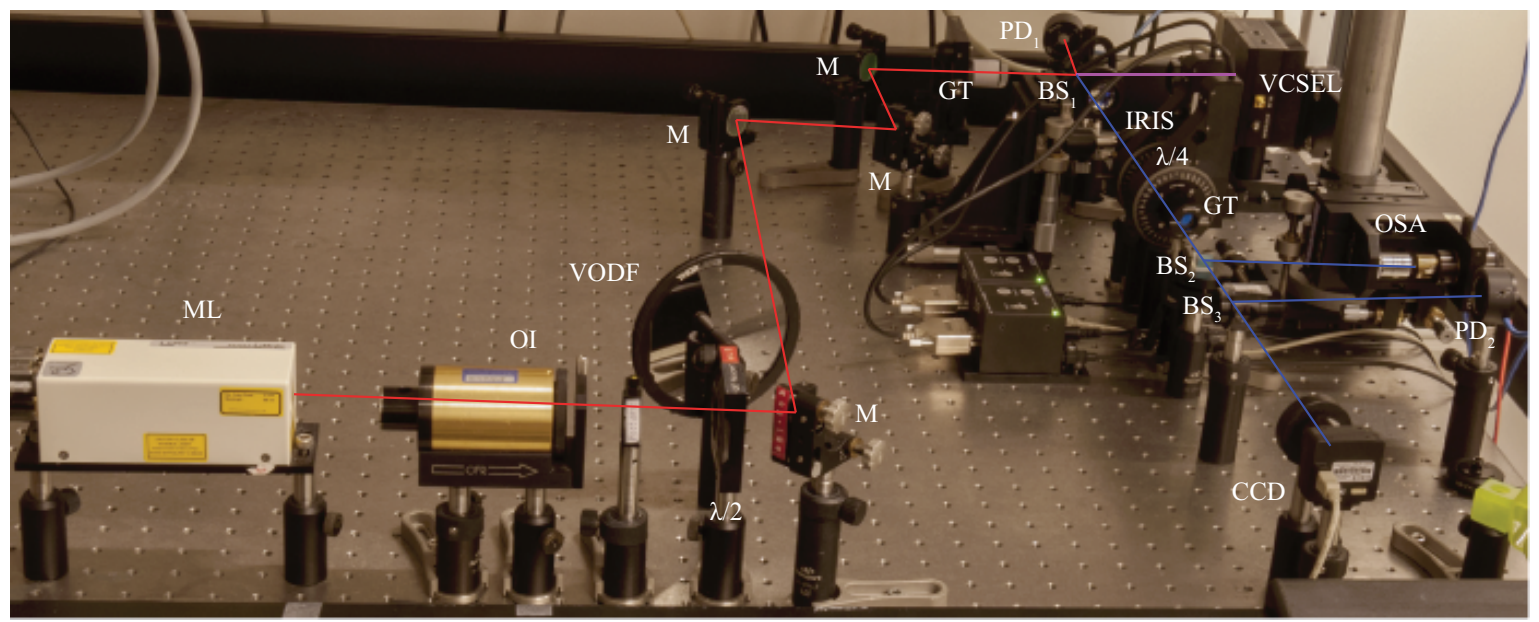

Figure 1. Picture of the experimental setup. The red line is the path of the light emitted by the Master Laser (depicted as ML on the picture), whereas the blue line is the path of light emitted from the VCSEL. OI: Optical Isolator. $\lambda / 2$ : Half Wave Plate. M: Mirror. VODF: Variable Optical Density Filter. GT: Glan Thompson prism. BS 1 : Beam Sampler. BS 2,3 : Beam Splitter. $\lambda / 4$ : Quarter Wave Plate. OSA: Optical Spectrum Analyzer. PD: Photodiode. CCD: CCD camera.

The aim of this paper is to further investigate this model, as it proved to be adapted to describe the polarization dynamics of CSs in VCSELs.

This communication is organised as follows: in the next section, we introduce a description of the experimental setup that has been used, alongside with the corresponding experimental data. In section 3, we introduce the VCSEL spin-flip model. In section 4, we perform a classification of different stability regimes. Finally, in section 5 , we conclude.

\section{EXPERIMENT}

The experimental setup is the one described in ${ }^{12}$ and in Fig. 1. The VCSEL is a $80 \mu \mathrm{m}$ diameter bottom-emitting multiple quantum well VCSEL similar to the ones described in. ${ }^{15}$ This VCSEL has a lasing threshold of $42.5 \mathrm{~mA}$ when kept at $25^{\circ} \mathrm{C}$. When being pumped close to its optical threshold, the light it emits is almost exclusively vertically polarized, ${ }^{16}$ and centered around $983.2 \mathrm{~nm}$. Its emission profile, when pumped slightly above threshold, has a particular structure which is often called daisy mode. In the first part of the experiment, our setup allows to record the power emitted by the VCSEL (using the photodiode marked $\mathrm{PD}_{2}$ in Fig. 1), its optical spectrum (using an Optical Spectrum Analyser ANDO AQ6317-B), and its near field profile (using a CCD camera).

The Master Laser (ML) is a commercial external cavity diode laser in Littrow configuration (Sacher Lasertechnik TEC-0960-060), isolated from the rest of the setup and whose power is monitored using the photodiode marked $\mathrm{PD}_{1}$ in Fig. 1. As its wavelength is tuned slightly above $983.2 \mathrm{~nm}$, it can lock the VCSEL. ${ }^{17}$ If one then tunes its optical power into the locking region (using either the half-wave plate $\lambda / 2$ for a fixed direction of the Glan-Thompson prism or using a Variable Optical Density Filter (VODF)), a bistability of the power emitted by the VCSEL as a function of the injection power can be recorded. It is worth noting that this bistability is associated with the appearing or disappearing of a localized spot in the near field profile of the VCSEL, which is a signature of a CS. An example thereof is shown in Fig. 2.

Once this experiment has been realized, and it has hence be proved that the bright dot appearing in the VCSEL near field is a CS indeed, the Stokes parameters of the CS can be measured. An iris is then set in the VCSEL path, to isolate the CS from the rest of the profile. A quarter-wave plate and a Glan-Thompson prism are used as well, in front of the photodiode $\mathrm{PD}_{2}$, to follow a textbook procedure of measuring Stokes parameters. ${ }^{18}$

The procedure can then be repeated for different directions of the linearly polarized optical injection. Results for this angle varying from 0 to $90^{\circ}$ are shown in Fig. 3. As $\Psi$ increases, one notices an evolution of the $\mathrm{s}_{1}$ and $\mathrm{s}_{2}$ parameters that tend to follow the optical injection linear polarization. However, as shown in Fig. 3c), the 


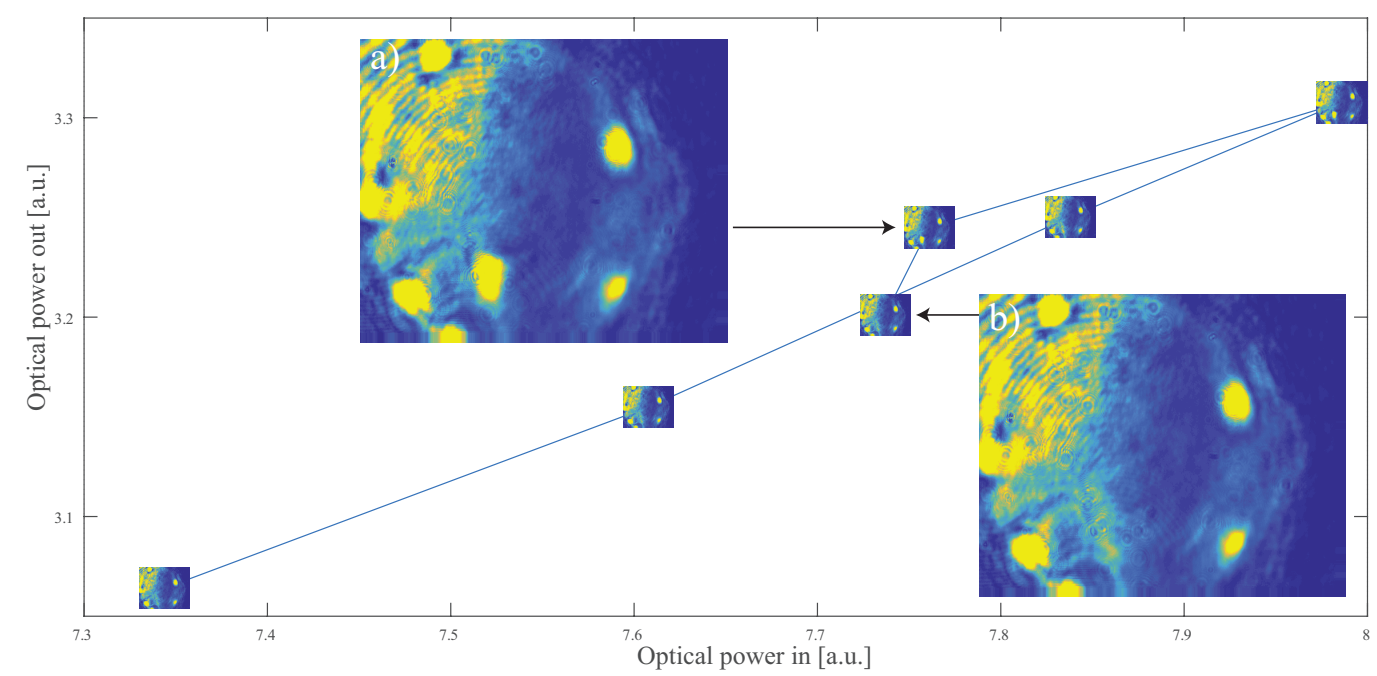

Figure 2. VCSEL output power as a function of injected power. Each measurement has been marked with its corresponding near-field profile. a) and b) are close ups of the marked data points. VCSEL current was set at 45.0mA, and it was kept at $25^{\circ} \mathrm{C}$.

linearly polarized part of the CS has a direction slightly different than the one of the optical injection. It is also worth noticing the clearly nonzero $\mathrm{s}_{3}$ in Fig. 3d), that demonstrates the non neglectible ellipticity of the CSs' polarization, and hence its vectorial nature.

\section{MODEL}

In order to describe the results presented in the former section, we consider, as in,${ }^{12}$ the spin-flip VCSEL model described in ${ }^{13,14}$ in which we have added diffraction. This model reads

$$
\begin{aligned}
\frac{\partial E_{x}}{\partial t} & =-\left(\kappa+\gamma_{a}\right) E_{x}-i\left(-\kappa \alpha+\gamma_{p}+\Delta \omega\right) E_{x}+\kappa(1-i \alpha)\left(N E_{x}+i n E_{y}\right)+\kappa E_{I} \cos (\Psi)+a i \nabla^{2} E_{x}, \\
\frac{\partial E_{y}}{\partial t} & =-\left(\kappa-\gamma_{a}\right) E_{y}-i\left(-\kappa \alpha-\gamma_{p}+\Delta \omega\right) E_{y}+\kappa(1-i \alpha)\left(N E_{y}-i n E_{x}\right)+\kappa E_{I} \sin (\Psi)+a i \nabla^{2} E_{y}, \\
\frac{\partial N}{\partial t} & =-\gamma\left[N\left(1+\left|E_{x}\right|^{2}+\left|E_{y}\right|^{2}\right)-\mu+i n\left(E_{y} E_{x}^{*}-E_{x} E_{y}^{*}\right)\right], \\
\frac{\partial n}{\partial t} & =-\gamma_{s} n-\gamma\left[n\left(\left|E_{x}\right|^{2}+\left|E_{y}\right|^{2}\right)+i N\left(E_{y} E_{x}^{*}-E_{x} E_{y}^{*}\right)\right] .
\end{aligned}
$$

In these equations, $E_{x}$ (resp. $E_{y}$ ) is the complex electric field envelope in the horizontal (resp. vertical) polarization direction. $\kappa$ is the photon lifetime, $\gamma_{a}$ is the VCSEL amplitude anisotropy, $\alpha$ is the linewidth enhancement factor and $\gamma_{p}$ is the VCSEL phase anistropy. $\Delta \omega$ is the frequency detuning between optical injection and VCSEL. $N+n$ is the number of carriers generating clockwise polarized light, whereas $N-n$ is the number of carriers generating counterclockwise polarized light. $\Psi$ is the angle between horizontal axis and optical injection linear polarization direction. $a$ is a scaling factor accounting for diffraction. The operator $\nabla^{2}$ acts in the $(x, y)$ plane. $\gamma$ is the carrier relaxation rate. $\mu$ accounts for current injection. $\gamma_{s}$ is the spin flip rate that accounts for relaxation of the carrier population difference $n$.

\section{ANALYSIS}

In this section, we investigate the different stability regimes of the equations 1-4 in the vicinity of the parameters that have been used in ${ }^{12}$ to describe the experimental results presented in this communication.

These parameters yield: $\alpha=3, \gamma=1 \mathrm{~ns}^{-1}, \gamma_{a}=0.1 \mathrm{~ns}^{-1}, \gamma_{p}=-20 \mathrm{~ns}^{-1}, \gamma_{s}=50 \mathrm{~ns}^{-1}, \kappa=200 \mathrm{~ns}^{-1}$ and $\mu=1.05$. Additionally, we consider an optical injection whose linear polarization makes an angle of $45^{\circ}$ with the horizontal axis. The homogeneous steady states of the system 1-4 have been computed numerically for $E_{I}$ 

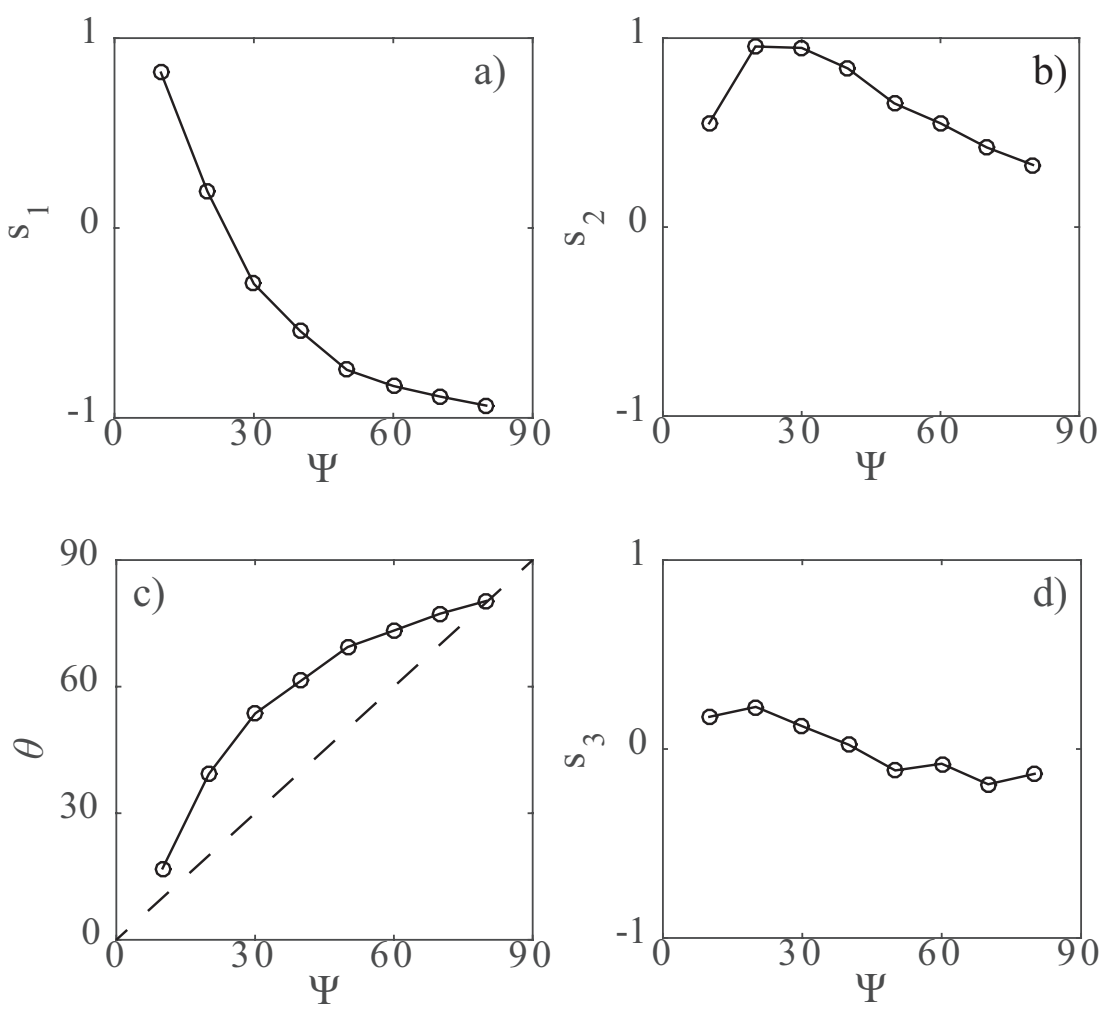

Figure 3. a)(b)): CS normalized $\mathrm{s}_{1}\left(\mathrm{~s}_{2}\right)$ parameter as a function of optical injection linear polarization angle with horizontal $\Psi$. c): connected circles: CS polarization main axis direction with respect with horizontal as a function of $\Psi$. Dashed line: $\theta=\Psi . \mathrm{d}$ ): CS normalized $\mathrm{s}_{3}$ parameter as a function of $\Psi$. Data are the same as the ones in. ${ }^{12}$

varying between 0.1 and 1 , and $\Delta \omega$ being set to 200,250 and $300 \mathrm{~ns}^{-1}$. The results of this analysis are displayed in Fig. 4.

The starting point for discussion is Fig. 4a). When the injection is zero, the homogeneous steady state of the system is unstable. When one gradually increases injection strength $E_{I}$, the unstable state begins to coexist with a Turing unstable state (point A). Very soon after a further increase of $E_{I}$, the unstable lower state becomes stable (point B), while the upper branch of the hysteresis stays Turing unstable. Further increasing the optical injection power leads to a disappearing of the lower branch of the hysteresis (point C). The upper Turing unstable branch finally becomes stable after some threshold optical injection power is reached (point D). In our parameter set, increasing the frequency detuning between the injection and the VCSEL (when moving from Fig. 4a) to Fig.4c)) has several effects:

1. It enlarges the transition threshold from a unstable state towards a stable state on the lower branch: the point B moves to the right.

2. It lowers the transition threshold from a Turing unstable state towards a stable state on the upper branch: the point $\mathrm{D}$ moves to the left.

3. It enlarges the transition threshold from a single state towards a bistable state, as well as the transition between a bistable state and a single state: points $\mathrm{A}$ and $\mathrm{C}$ move to the right.

4. The width of the hysteresis loop is also slightly enlarged: the distance between points A and C increases.

This analysis allows to find several parameter sets for finding CSs. Indeed, when a Turing unstable state coexists with a stable state, CSs can be generated inside the system. This is illustrated in Fig. 5, that is a numerical simulation of Eqs. 1-4 with the parameters of Fig. 4c) and $E_{I}=0.5$, expressed in terms of Stokes parameters $\left(S_{0}, S_{1}, S_{2}, S_{3}\right)$ and populations $(N, n)$. 

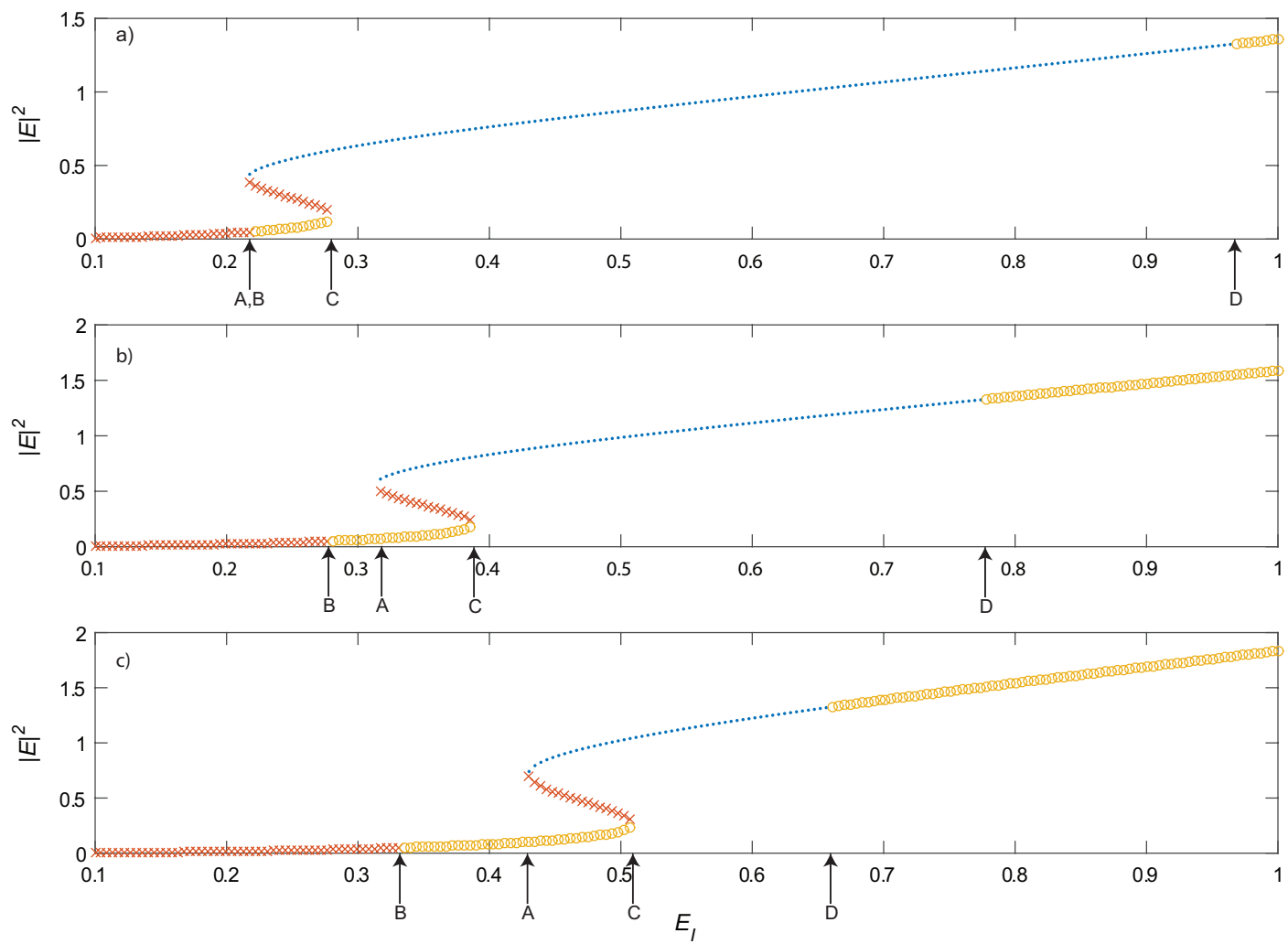

Figure 4. Stability regimes of Eqs. 1-4 as a function of $E_{I}$ for $\Delta \omega=200$ (a), 250(b) and $300 \mathrm{~ns}^{-1}$ (c). Parameters are $\alpha=3, \gamma=1 \mathrm{~ns}^{-1}, \gamma_{a}=0.1 \mathrm{~ns}^{-1}, \gamma_{p}=-20 \mathrm{~ns}^{-1}, \gamma_{s}=50 \mathrm{~ns}^{-1}, \kappa=200 \mathrm{~ns}^{-1}$ and $\mu=1.05$. Crosses denote plane-wave unstable states, circles denote stable states, and dots represent Turing unstable states.
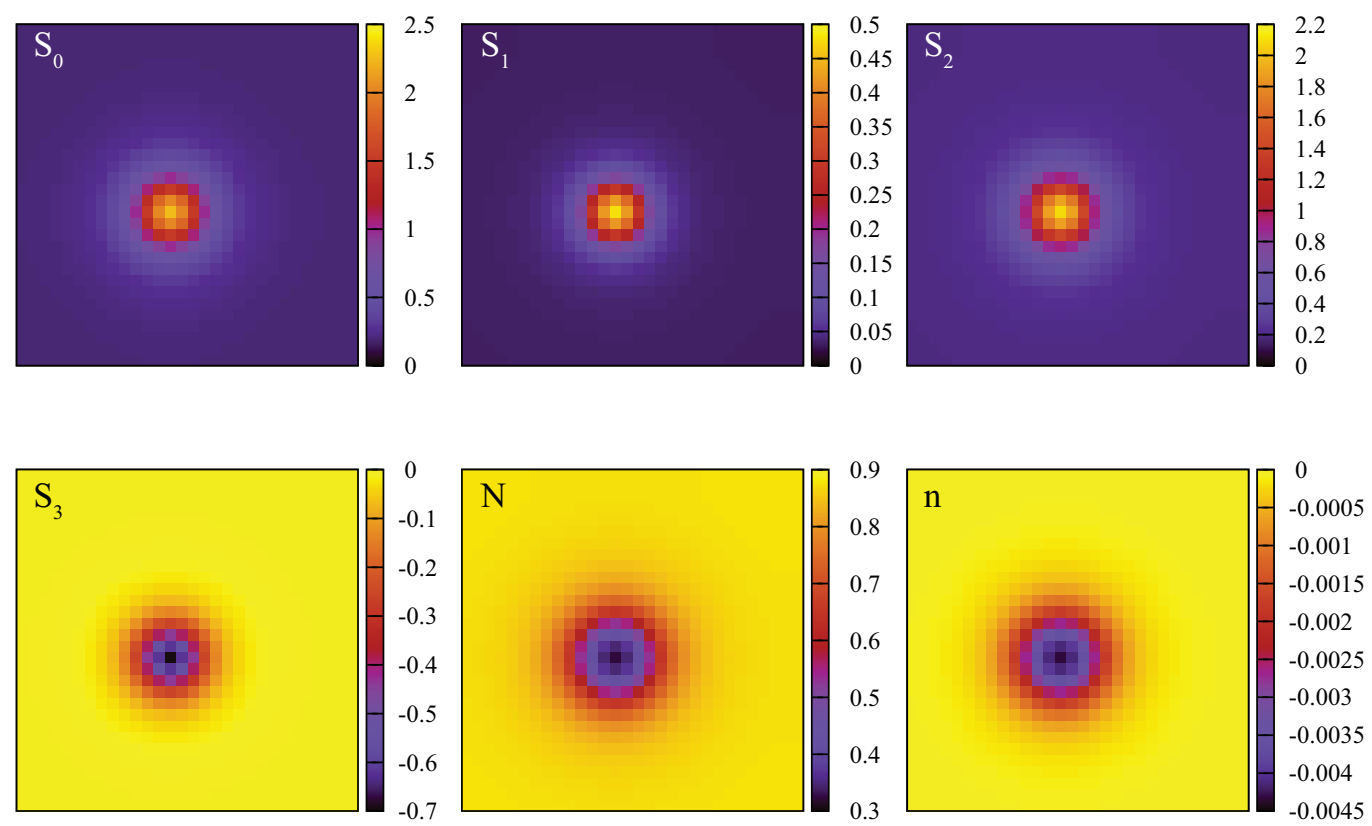

Figure 5. Numerical simulation of Eqs. 1-4. Parameters are the same as in Fig. 4c) and $E_{I}=0.5$. 


\section{CONCLUSION}

In this communication, we presented experimental evidence of localized structures having multiple polarization components. We also presented a model to describe these structures. We performed a linear stability analysis of this model, for different experimentally relevant parameters. We commented on the evolution of the stability domains with changing the value of a single parameter, namely the detuning between optical injection and laser frequency.

This study is important for accessing the experimentally relevant parameters of the optically injected VCSEL for the observation of vector CSs.

\section{ACKNOWLEDGMENTS}

M.T. received support from the Fonds National de la Recherche Scientifique (Belgium). This research was partially supported by EU FP7 ICT FET Open HIDEAS and by the Interuniversity Attraction Poles program of the Belgian Science Policy Office, under grant IAP P7-35 photonics@be.

\section{REFERENCES}

[1] Schulz-Ruhtenberg, M., Tanguy, Y., Jäger, R., and Ackemann, T., "Length scales and polarization properties of annular standing waves in circular broad-area vertical-cavity surface-emitting lasers," Applied Physics $B$ 97(2), 397-403 (2009).

[2] Panajotov, K., Danckaert, J., Verschaffelt, G., Peeters, M., Nagler, B., Albert, J., Ryvkin, B., Thienpont, H., and Veretennicoff, I., "Polarization behavior of vertical-cavity surface-emitting lasers: Experiments, models and applications," AIP Conference Proceedings 560(1), 403-417 (2001).

[3] Panajotov, K. and Prati, F., "Polarization dynamics of vcsels," in [VCSELs], Michalzik, R., ed., Springer Series in Optical Sciences 166, ch. 6, 181-231, Springer Berlin Heidelberg (2013).

[4] Hachair, X., Tissoni, G., Thienpont, H., and Panajotov, K., "Linearly polarized bistable localized structure in medium-size vertical-cavity surface-emitting lasers," Phys. Rev. A 79, 011801 (Jan 2009).

[5] Leblond, H. and Mihalache, D., "Models of few optical cycle solitons beyond the slowly varying envelope approximation," Physics Reports 523(2), 61 - 126 (2013).

[6] Tlidi, M., Staliunas, K., Panajotov, K., Vladimirov, A. G., and Clerc, M. G., "Localized structures in dissipative media: from optics to plant ecology," Philosophical Transactions of the Royal Society of London A: Mathematical, Physical and Engineering Sciences 372, 20140101 (2014).

[7] Lugiato, L., Prati, F., and Brambilla, M., [Nonlinear Optical Systems], Cambridge University Press (2015).

[8] Tlidi, M., Mandel, P., and Lefever, R., "Localized structures and localized patterns in optical bistability," Phys. Rev. Lett. 73, 640-643 (Aug 1994).

[9] Scroggie, A. J., Firth, W. J., McDonald, G. S., Tlidi, M., Lefever, R., and Lugiato, L. A., "Pattern formation in a passive kerr cavity," Chaos, Solitons and Fractals 4(8), 1323 (1994).

[10] Firth, W. J. and Scroggie, A. J., "Optical bullet holes: Robust controllable localized states of a nonlinear cavity," Phys. Rev. Lett. 76, 1623 (Mar 1996).

[11] Barland, S., Tredicce, J. R., Brambilla, M., Lugiato, L. A., Balle, S., Giudici, M., Maggipinto, T., Spinelli, L., Tissoni, G., Knödl, T., et al., "Cavity solitons as pixels in semiconductor microcavities," Nature 419(6908), 699 (2002).

[12] Averlant, E., Tlidi, M., Thienpont, H., Ackemann, T., and Panajotov, K., "Vector cavity solitons in broad area vertical-cavity surface-emitting lasers," Scientific Reports 6, 20428 (Feb. 2016).

[13] San Miguel, M., Feng, Q., and Moloney, J. V., "Light-polarization dynamics in surface-emitting semiconductor lasers," Phys. Rev. A 52, 1728-1739 (Aug 1995).

[14] Martin-Regalado, J., Prati, F., San Miguel, M., and Abraham, N., "Polarization properties of vertical-cavity surface-emitting lasers," Quantum Electronics, IEEE Journal of 33, 765-783 (May 1997).

[15] Grabherr, M., Miller, M., Jager, R., Michalzik, R., Martin, U., Unold, H. J., and Ebeling, K. J., "Highpower vcsels: single devices and densely packed 2-d-arrays," Selected Topics in Quantum Electronics, IEEE Journal of $\mathbf{5}(3), 495$ (1999). 
[16] Averlant, E., Tlidi, M., Thienpont, H., Ackemann, T., and Panajotov, K., "Experimental observation of localized structures in medium size vcsels," Opt. Express 22, 762-772 (Jan 2014).

[17] van Tartwijk, G. H. M. and Lenstra, D., "Semiconductor lasers with optical injection and feedback," Quantum and Semiclassical Optics: Journal of the European Optical Society Part B 7(2), 87 (1995).

[18] Born, M. and Wolf, E., [Principles of Optics], Cambridge University Press, 7th ed. (1999). 